\title{
Correlation between hs-CRP, IL-6, IL-10, ET-1, and Chronic Obstructive Pulmonary Disease Combined with Pulmonary Hypertension
}

\author{
Danfen Yang $\mathbb{D}^{1},{ }^{1}$ Li Wang $\mathbb{D}^{2},{ }^{2}$ Pengfei Jiang $\mathbb{D},{ }^{3}$ Rui Kang $\mathbb{D},{ }^{2}$ and Yuanyuan Xie $\mathbb{D}^{1}$ \\ ${ }^{1}$ Department of Geriatrics, Affiliated Hospital of Yan'an University, Yan'an 716000, Shaanxi, China \\ ${ }^{2}$ Department of Respiratory, Affiliated Hospital of Yan'an University, Yan'an 716000, Shaanxi, China \\ ${ }^{3}$ Department of Orthopedics, Affiliated Hospital of Yan'an University, Yan'an 716000, Shaanxi, China
}

Correspondence should be addressed to Yuanyuan Xie; xyy@yau.edu.cn

Received 17 December 2021; Accepted 29 December 2021; Published 10 February 2022

Academic Editor: Kalidoss Rajakani

Copyright ( 2022 Danfen Yang et al. This is an open access article distributed under the Creative Commons Attribution License, which permits unrestricted use, distribution, and reproduction in any medium, provided the original work is properly cited.

With the development of society, chronic obstructive pulmonary disease (COPD), a common respiratory disease, suffers an increasing incidence. To explore the correlation between high-sensitivity C-reactive protein (hs-CRP), interleukin-6 (IL-6), interleukin-10 (IL-10), endothelin-1 (ET-1), and chronic obstructive pulmonary disease combined with pulmonary hypertension (COPD-PH), a total of 112 COPD patients admitted to our hospital from July 2017 to December 2020 were analyzed prospectively, of which 57 patients combined with $\mathrm{PH}$ were enrolled in the research group and the other 55 patients without $\mathrm{PH}$ were enrolled in the control group. Serum hs-CRP, IL-6, IL-10, ET-1, blood gas indexes, and related indexes of lung function of the two groups were detected and their correlations were analyzed. The research group was divided into the mild group, moderate group, and heavy group according to pulmonary average arterial pressure, and serum hs-CRP, IL-6, IL-10, ET-1, and disease severity were analyzed. Receiver operating characteristic curve (ROC) analysis was performed to serum hs-CRP, IL-6, IL-10, and ET-1 of COPD-PH patients, and independent risk factors for COPD-PH were analyzed. The research group showed significantly higher serum hs-CRP, IL-6, and ET-1 and significantly lower IL-10 expression than the control group (all $P<0.05$ ); serum hs-CRP, IL-6, and ET-1 were negatively correlated with PaO2, FEV1, FVC, and FEV1/FVC and positively correlated with PaCO2; IL-10 was positively correlated with PaO2, FEV1, FVC, and FEV1/FVC and negatively correlated with PaCO2; hs-CRP, IL-6, and ET-1 were positively correlated with COPD-PH severity, and IL-10 was negatively correlated with it. hs-CRP, IL-6, IL-10, and ET-1 were closely and significantly related to the pathological process of COPD-PH, including onset and development, and the elevation of hs-CRP, IL-6, and ET-1 and decrease of IL-10 are independent risk factors for the onset of COPD-PH. With relatively high predictive value for COPD-PH, hs-CRP, IL-6, IL-10, and ET-1 can be promoted as predictors for it.

\section{Introduction}

With the development of society, chronic obstructive pulmonary disease (COPD), a common respiratory disease, suffers an increasing incidence [1]. Pulmonary hypertension $(\mathrm{PH})$, one of the main complications of COPD, is a disease for pulmonary vascular endothelial function disorder and pulmonary vascular remodeling due to hypoxic pulmonary vasoconstriction for repeated acute disease exacerbation of patients, and it is also one of the important factors leading to poor prognosis of COPD patients [2,3]. A study for secondary
PH indicated that systemic inflammation and hypoxia play an important role in the pathogenesis of $\mathrm{PH}$ [4].

In recent years, the role of inflammatory response in the secondary PH of COPD has drawn much attention, and it is believed that a variety of inflammatory factors play an important role in the regulation of pulmonary artery pressure [5, 6]. High-sensitivity C-reactive protein (hs-CRP) is an acute reactive protein synthesized by the liver. A study pointed out that hs-CRP is an important index of systemic inflammatory response, and its expression level will not be affected by cortical hormone treatment [7]. As relatively 
representative inflammatory factors, interleukin-6 (IL-6) and interleukin-10 (IL-10) also play an important role in the pathogenesis of $\mathrm{PH}$ [8]. Endothelin is a factor proven to be with an important function in pulmonary vascular resistance increase and pulmonary vascular remodeling, and endothelin-1 (ET-1) is one of the most important factors of it [9]. $\mathrm{PH}$ is a disease characterized by progressive rising of pulmonary vascular resistance. When COPD causes airway obstruction and damages pulmonary parenchyma and pulmonary vasculature, pulmonary arterial endothelium will suffer hyperplasia and fibrosis, eventually leading to pulmonary circulation remodeling and onset of $\mathrm{PH}$. A study pointed out that ET-1 expression has a very close relationship with the degree of distal pulmonary vascular damage [10]. This study aims to explore the correlation between high-sensitivity C-reactive protein (hs-CRP), interleukin-6 (IL-6), interleukin-10 (IL-10), endothelin-1 (ET1 ), and chronic obstructive pulmonary disease combined with pulmonary hypertension (COPD-PH).

Therefore, this study explored the correlation between hs-CRP, IL-6, IL-10, ET-1, and COPD-PH, so as to provide a more theoretical basis for early intervention and treatment for COPD-PH patients.

\section{Materials and Methods}

2.1. General Materials. A total of 112 COPD patients (65.21 \pm 4.77 years old in mean age) admitted to our hospital from July 2015 to December 2018 were analyzed prospectively, of which 57 patients combined with $\mathrm{PH}$ were enrolled in the research group and other 55 patients without $\mathrm{PH}$ were enrolled in the control group. Inclusion criteria: patients meeting the diagnostic criteria for COPD and patients (to be enrolled in the research group) meeting the diagnostic criteria in 2009 European Pulmonary Hypertension Guidelines. Exclusion criteria: patients with other complications, primary diseases of lungs, severe hepatic renal dysfunction, other malignant tumors or severe immune system disease, patients with communication obstacles and cognitive impairment, and patients unwilling to cooperate for the experiment. The experiment has been approved by the ethics committee of our hospital, and all patients and their families agreed to participate in the experiment and signed an informed consent form.

\subsection{Related Index Detection}

2.2.1. Blood Gas Index and Related Inflammatory Factor Detection. Blood gas analysis was performed on all patients after admission (determined by an ABL700 blood gas analyzer from Danish Radiometer Medical Equipment Co., Ltd.), and $5 \mathrm{ml}$ of fasting venous blood taken in the next morning after admission was centrifuged at $3000 \mathrm{r} / \mathrm{min}$ and stored at $-80^{\circ} \mathrm{C}$ for later detection. hs-CRP was determined by an automatic biochemistry analyzer through immune turbidimetry, and IL-6, IL-10, and ET-1 were determined by ELISA (IL-6 and IL-10 ELISA kits were purchased from Shanghai Jining Industrial Biotechnology Co., Ltd., N110863, N110820; ET-1 kit was purchased from Beijing Reanta Biotechnology Co., Ltd., YAD) in strict accordance with kit instructions.

2.2.2. Pulmonary Function Detection. Pulmonary function indexes were detected for all patients after admission based on the premise that the patients have not received bronchodilator or glucocorticoid drugs within 48 hours before pulmonary function detection, which mainly included the detection and calculation of forced expiratory volume in one second (FEV1), forced vital capacity (FVC), and FEV1/FVC of patients.

2.2.3. Pulmonary Artery Pressure Determination. All patients were determined in pulmonary artery pressure through a color doppler echocardiography in a peaceful supine position, and the four chamber images of their apical muscular were taken based on the premises of keeping sample lines parallel to regurgitation. The patients' $\mathrm{PH}$ was calculated according to the Bernoulli simplified formula: PASP $=4 \mathrm{~V} 2$ (maximum tricuspid regurgitation rate) $+5 \mathrm{mmHg}[11]$.

2.2.4. Statistical Analysis. In the study, SPSS 19.0 software (Bizinsight (Beijing) Information Technology Co., Ltd.) was adopted for statistical analysis of experiment data; count data were analyzed by the chi-square test, and measurement data were expressed in mean \pm standard deviation. Comparison between the two groups was tested by $t$, and their correlations were analyzed using the Pearson correlation coefficient. Logistic multivariate analysis was performed for risk factors of $\mathrm{PH} . P<0.05$ indicated statistical difference.

\section{Results}

3.1. Comparison in General Materials. There were no significant differences in gender, age, and body mass index (BMI) between the two groups (all $P>0.05$ ). More details are given in Table 1.

3.2. Comparison between the Two Groups in Serum hs-CRP, IL-6, IL-10, and ET-1 Expression. The research group showed significantly higher serum hs-CRP, IL-6, and ET-1 and significantly lower IL-10 expression than the control group (all $P<0.05$ ). More details are given in Table 2 .

3.3. Related Blood Gas Index Detection of the Two Groups. In terms of blood gas index, the research group showed significantly lower $\mathrm{PaO} 2$ and significantly higher $\mathrm{PaCO} 2$ than the control group (both $P<0.05$ ). More details are given in Table 3 .

3.4. Pulmonary Function Indexes and Pulmonary Average Arterial Pressure of the Two Groups. The research group showed significantly lower FEV1, FVC, and FEV1/FVC and significantly higher pulmonary average arterial pressure than the control group (all $P<0.05$ ). More details are given in Table 4. 
TABle 1: General condition ( $n(\%))$.

\begin{tabular}{|c|c|c|c|c|}
\hline Factor & The research group, $n=57$ & The control group, $n=55$ & $t / \chi^{2}$ & $P$ \\
\hline Gender & & & 0.002 & 0.964 \\
\hline Male & $35(61.40)$ & $34(61.82)$ & & \\
\hline Female & $22(38.60)$ & $21(38.18)$ & & \\
\hline Age $(Y)$ & & & 0.001 & 0.975 \\
\hline$\leq 65$ & $24(42.10)$ & $23(41.82)$ & & \\
\hline$>65$ & $33(57.90)$ & $32(58.18)$ & & \\
\hline BMI $\left(\mathrm{kg} / \mathrm{m}^{2}\right)$ & & & 0.000 & 0.992 \\
\hline$\leq 22$ & $27(47.37)$ & $26(47.27)$ & & \\
\hline$>22$ & $30(52.63)$ & $29(52.73)$ & & \\
\hline Smoking history & & & 0.007 & 0.932 \\
\hline Yes & $40(70.18)$ & $39(70.91)$ & & \\
\hline No & $17(29.82)$ & $16(29.09)$ & & \\
\hline Course of disease & $4.31 \pm 2.21$ & $4.29 \pm 2.19$ & 0.049 & 0.961 \\
\hline \multicolumn{5}{|l|}{ Coagulation function } \\
\hline APTTs & $27.91 \pm 2.02$ & $28.05 \pm 2.04$ & 0.368 & 0.713 \\
\hline PTs & $11.54 \pm 1.23$ & $11.57 \pm 1.21$ & 0.131 & 0.896 \\
\hline FIB g/l & $3.15 \pm 0.14$ & $3.16 \pm 0.13$ & 0.395 & 0.694 \\
\hline \multicolumn{5}{|l|}{ Education level } \\
\hline With junior high school diploma and below & $29(50.88)$ & $28(50.91)$ & 0.000 & 0.997 \\
\hline With junior high school diploma and above & $28(49.12)$ & $27(49.09)$ & & \\
\hline
\end{tabular}

TABLE 2: Comparison between the two groups in serum hs-CRP, IL-6, IL-10, and ET-1 expression.

\begin{tabular}{lcrr}
\hline Factor & The research group, $n=57$ & The control group, $n=55$ & \multicolumn{1}{c}{$P$} \\
\hline hs-CRP $(\mathrm{mg} / \mathrm{L})$ & $42.76 \pm 3.54$ & $37.27 \pm 1.37$ & 10.75 \\
IL-6 $(\mathrm{pg} / \mathrm{mL})$ & $104.68 \pm 8.35$ & $93.12 \pm 6.32$ & 8.239 \\
IL-10 $(\mathrm{pg} / \mathrm{mL})$ & $4.38 \pm 0.45$ & $5.23 \pm 0.37$ & $<.001$ \\
ET-1 $(\mathrm{umol} / \mathrm{L})$ & $43.91 \pm 9.94$ & $30.18 \pm 7.59$ & $<0.001$ \\
\hline
\end{tabular}

TABLE 3: Related blood gas index detection of the two groups.

\begin{tabular}{lccr}
\hline Factor & The research group, $n=57$ & The control group, $n=55$ & $t$ \\
\hline $\mathrm{PaO} 2(\mathrm{mmHg})$ & $69.26 \pm 8.19$ & $95.19 \pm 10.65$ & 14.47 \\
$\mathrm{PaCO} 2(\mathrm{mmHg})$ & $56.39 \pm 9.28$ & $43.19 \pm 7.56$ & 8.236 \\
\hline
\end{tabular}

TABLE 4: Pulmonary function indexes and pulmonary average arterial pressure of the two groups.

\begin{tabular}{|c|c|c|c|c|}
\hline Factor & The research group, $n=57$ & The control group, $n=55$ & $t$ & $P$ \\
\hline FEV1 (\%) & $50.98 \pm 4.18$ & $64.51 \pm 5.39$ & 14.88 & $<0.001$ \\
\hline FVC (\%) & $63.71 \pm 4.26$ & $72.23 \pm 6.51$ & 8.224 & $<0.001$ \\
\hline FEV1/FVC & $51.19 \pm 5.54$ & $57.71 \pm 6.37$ & 5.786 & $<0.001$ \\
\hline Pulmonary average arterial pressure $(\mathrm{mmHg})$ & $59.61 \pm 15.35$ & $24.19 \pm 5.47$ & 34.64 & $<0.001$ \\
\hline
\end{tabular}

3.5. Correlation Analysis of Serum hs-CRP, IL-6, IL-10, ET-1, and Blood Gas Indexes. Serum hs-CRP, IL-6, and ET-1 were negatively correlated with $\mathrm{PaO} 2(P<0.001)$ and positively correlated with $\mathrm{PaCO} 2(P<0.001)$. IL-10 was positively correlated with $\mathrm{PaO} 2(P<0.001)$ and negatively correlated with $\mathrm{PaCO} 2(P<0.001)$. More details are given in Table 5 and Figure 1.

3.6. Correlation Analysis of Serum hs-CRP, IL-6, IL-10, ET-1, and Pulmonary Function Indexes. Serum hs-CRP, IL-6, and ET-1 were negatively correlated with FEV1, FVC, and FEV1/ FVC (all $P<0.05$ ), and ET-1 was positively correlated with
FEV1, FVC, and FEV1/FVC (all $P<0.05$ ). More details are given in Table 6 and Figure 2.

3.7. Correlation Analysis of Serum hs-CRP, IL-6, IL-10, ET-1, and Severity of COPD. According to pulmonary average arterial pressure, the patients were divided into the mild group (40-50 $\mathrm{mmHg}, 17$ patients), moderate group (50-65 mmHg, 24 patients), and heavy group (>65 $\mathrm{mmHg}$, 16 patients). Comparison showed that the more severe the patients' disease, the higher the patients' hs-CRP, IL-6, and ET-1 expression and the lower the IL-10 expression. The correlation was statistically significant $(P<0.05)$. More 
TABLE 5: Correlation analysis of serum hs-CRP, IL-6, IL-10, ET-1, and blood gas indexes.

\begin{tabular}{lcccc}
\hline Index & & $\mathrm{PaO} 2$ & \multicolumn{2}{c}{ PaCO2 } \\
\hline hs-CRP & $r$ & $P$ & \multicolumn{1}{c}{ P } & $<0001$ \\
IL-6 & -0.776 & $<0.001$ & 0.871 & $<0.001$ \\
IL-10 & -0.786 & $<0.001$ & -0.617 & $<0.001$ \\
ET-1 & 0.594 & $<0.001$ & 0.797 & $<0.001$ \\
\hline
\end{tabular}

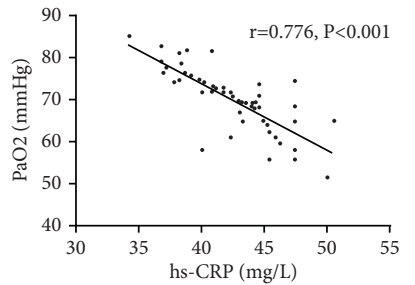

(a)

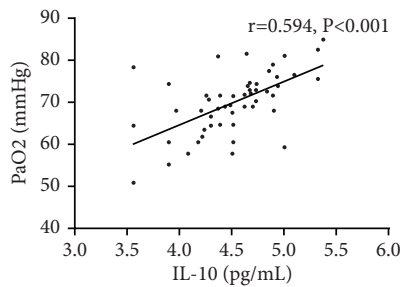

(e)

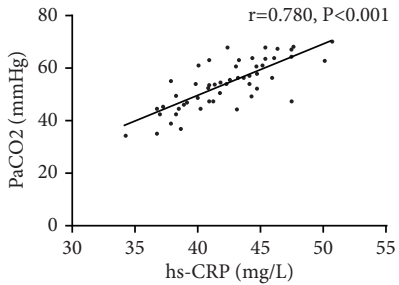

(b)

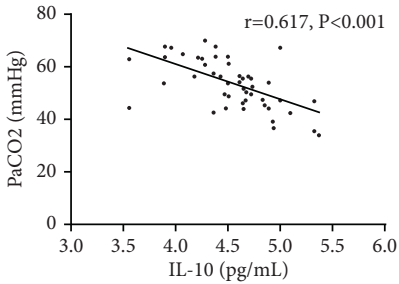

(f)

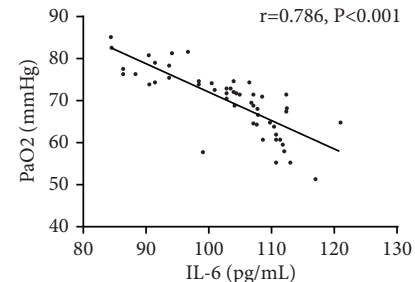

(c)

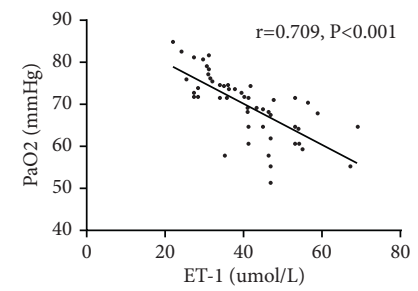

(g)

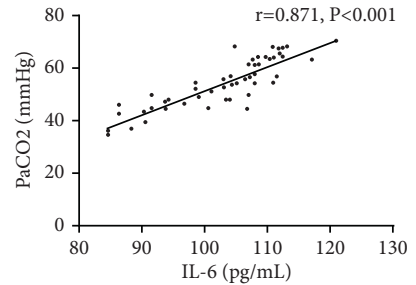

(d)

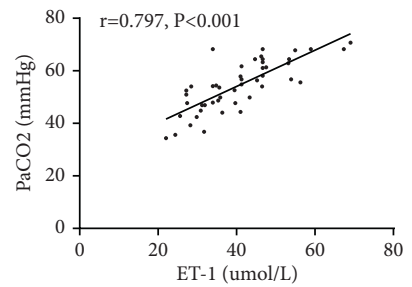

(h)

Figure 1: Correlation analysis of serum hs-CRP, IL-6, IL-10, ET-1, and blood gas indexes. (a) Negative correlation between hs-CRP and $\mathrm{PaO} 2(r=-0.776, P<0.001)$. (b) Positive correlation between hs-CRP and PaCO2 $(r=0.780, P<0.001)$. (c) Negative correlation between IL-6 and PaO2 $(r=-0.786, P<0.001)$. (d) Positive correlation between IL-6 and PaCO2 $(r=0.871, P<0.001)$. (e) Positive correlation between IL-10 and $\mathrm{PaO} 2(r=0.594, P<0.001)$. (f) Negative correlation between IL-10 and $\mathrm{PaO} 2(r=-0.709, P<0.001)$. (g) Negative correlation between ET-1 and PaO2 $(r=-0.709, P<0.001)$. (h) Positive correlation between ET-1 and PaCO2 $(r=0.797$, $P<0.001)$.

TABLE 6: Correlation analysis of serum hs-CRP, IL-6, IL-10, ET-1, and pulmonary function indexes.

\begin{tabular}{|c|c|c|c|c|c|c|}
\hline \multirow{2}{*}{ Index } & \multicolumn{2}{|c|}{ FEV1 } & \multicolumn{2}{|c|}{ FVC } & \multicolumn{2}{|c|}{ FEV1/FVC } \\
\hline & $r$ & $P$ & $r$ & $P$ & $r$ & $P$ \\
\hline hs-CRP & -0.725 & $<0.001$ & -0.759 & $<0.001$ & -0.808 & $<0.001$ \\
\hline IL-6 & -0.783 & $<0.001$ & -0.835 & $<0.001$ & -0.811 & $<0.001$ \\
\hline IL-10 & 0.520 & $<0.001$ & 0.601 & $<0.001$ & 0.580 & $<0.001$ \\
\hline ET-1 & -0.816 & $<0.001$ & -0.785 & $<0.001$ & -0.701 & $<0.001$ \\
\hline
\end{tabular}

details are given in Table 7 . In addition, correlation analysis showed that the severity of the disease was positively correlated with hs-CRP, IL-6, and ET-1 $(r=0.808, P<0.05$; $r=0.883, P<0.03 ; r=0.817, P<0.05)$ and negatively correlated with IL-1 $(r=-0.597, P<0.05)$. More details are shown in Figure 3.

3.8. ROC Analysis of hs-CRP, IL-6, IL-10, and ET-1 to COPD$P H$. In terms of COPD-PH diagnosis, hs-CRP showed sensitivity of $90.91 \%$, specificity of $85.96 \%$, area-under-thecurve (AUG) of 0.899 , and critical value of 38.49 (mg/L); IL6 showed sensitivity of $87.27 \%$, specificity of $89.47 \%$, AUG of 0.929 , and critical value of $98.99(\mathrm{pg} / \mathrm{mL})$; IL-10 showed sensitivity of $81.82 \%$, specificity of $68.42 \%$, AUG of 0.854 , and critical value of $4.740(\mathrm{pg} / \mathrm{mL})$, and ET-1 showed sensitivity of $87.27 \%$, specificity of $57.89 \%$, AUG of 0.805 , and critical value of $39.64(\mathrm{pg} / \mathrm{mL})$. More details are shown in Figure 4.

3.9. Multivariate Analysis of the Incidence of $\mathrm{PH}$. The above study indicated that elevation of hs-CRP, IL-6, and ET-1 and decrease of IL-10 were correlated with COPD-PH. Assignment was done to those factors (Table 8), and logistic regression analysis was performed to analyze whether those factors were independent risk factors for the onset of COPD-PH, and it turned out that elevation of hs-CRP (OR: 2.314, 95\% CI: 1.104-4.211), IL-6 (OR: 2.564, 95\% CI: 1.114-4.789), and ET-1 (OR: 3.759, 95\% CI: 1.229-5.116) and decrease of IL-10 (OR: 6.151, 95\% CI: 3.119-5.243) were all independent risk factors for the onset of COPD-PH. More details are given in Table 9. 


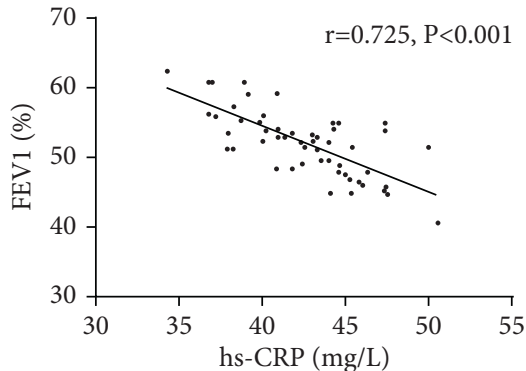

(a)

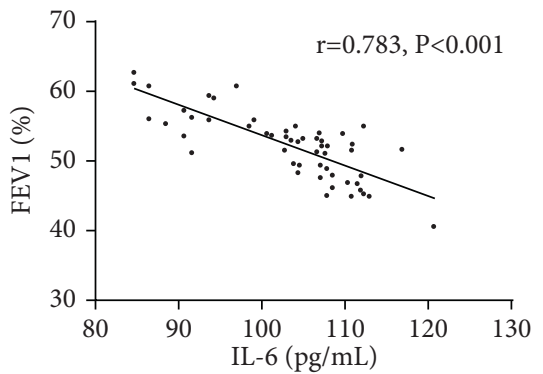

(d)

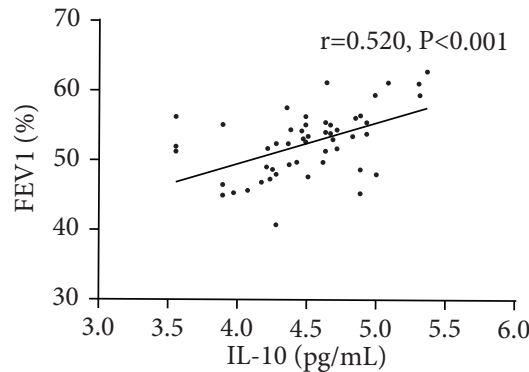

(g)

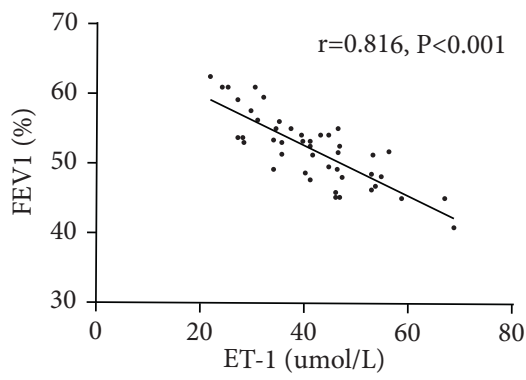

(j)

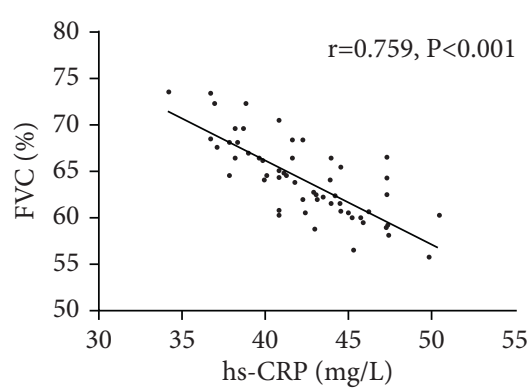

(b)

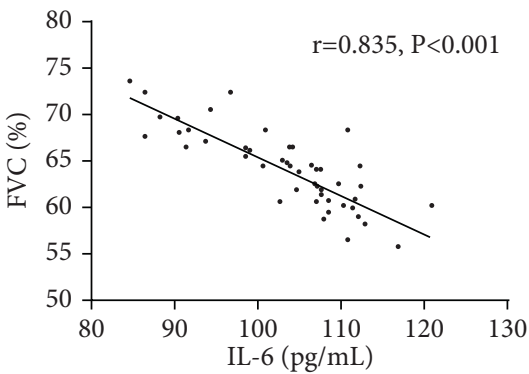

(e)

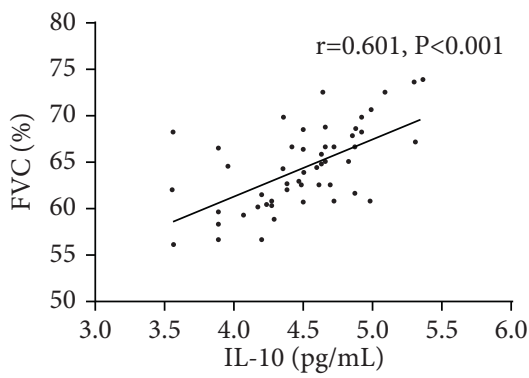

(h)

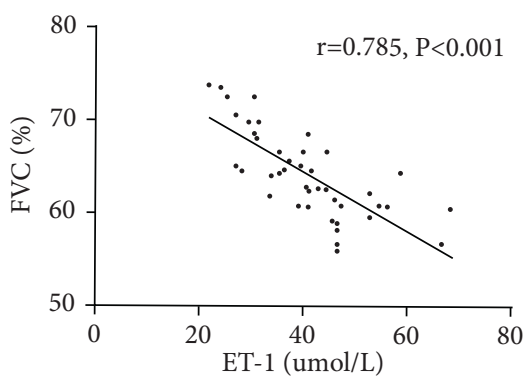

(k)

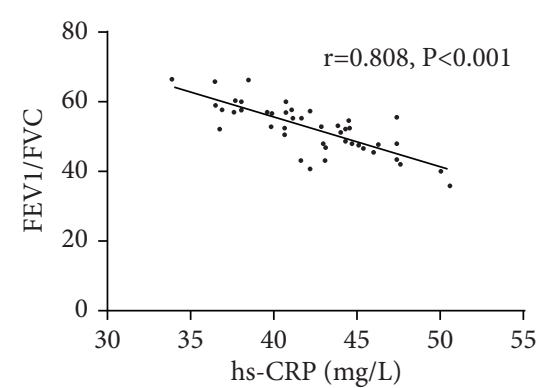

(c)

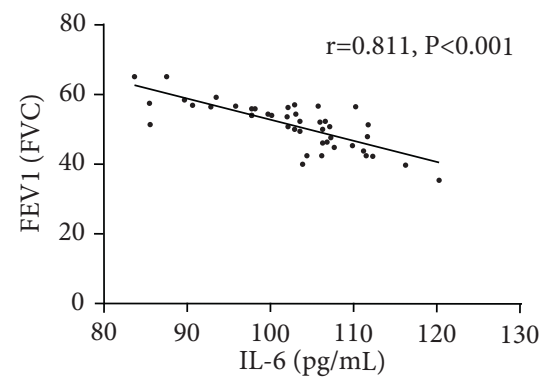

(f)

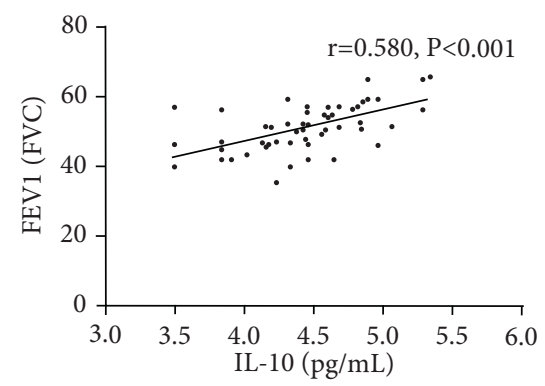

(i)

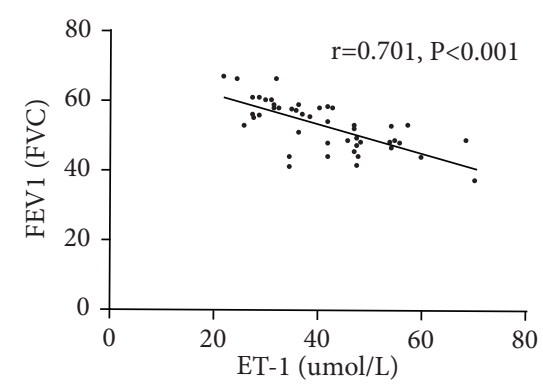

(l)

FIgURE 2: Correlation analysis of serum hs-CRP, IL-6, IL-10, ET-1, and pulmonary function indexes. (a) Negative correlation between hs-CRP and FEV1 $(r=-0.725, P<0.001)$. (b) Negative correlation between hs-CRP and FVC $(r=-0.759, P<0.001)$. (c) Negative correlation between hs-CRP and FEV1/FVC $(r=-0.808, P<0.001)$. (d) Negative correlation between IL-6 and FEV1 $(r=-0.783$, $P<0.001)$. (e) Negative correlation between IL-6 and FVC $(r=-0.835, P<0.001)$. (f) Negative correlation between IL-6 and FEV1/FVC $(r=-0.811, P<0.001)$. (g) Positive correlation between IL-10 and FEV1 $(r=0.520, P<0.001)$. (h) Positive correlation between IL-10 and FVC $(r=0.601, P<0.001)$. (i) Positive correlation between IL-10 and FEV1/FVC $(r=0.580, P<0.001)$. (j) Negative correlation between ET-1 and FEV1 $(r=-0.816, P<0.001)$. (k) Negative correlation between ET-1 and FVC $(r=-0.785, P<0.001)$. (l) Negative correlation between ET-1 and FEV1/FVC $(r=-0.701, P<0.001)$. 
TABLE 7: Blood gases hs-CRP, IL-6, IL-10, and ET-1 expression in patients with different severities of disease in the research group.

\begin{tabular}{|c|c|c|c|c|c|}
\hline Factor & The mild group, $n=17$ & The moderate group, $n=24$ & The heavy group, $n=16$ & $F$ & $P$ \\
\hline hs-CRP (mg/L) & $38.51 \pm 1.01$ & $42.76 \pm 2.19$ & $45.76 \pm 3.54$ & 37.83 & $<0.001$ \\
\hline IL-6 (pg/mL) & $99.29 \pm 5.13$ & $105.11 \pm 6.13$ & $109.91 \pm 7.19$ & 17.15 & $<0.001$ \\
\hline IL-10 (pg/mL) & $4.88 \pm 0.45$ & $4.43 \pm 0.43$ & $3.92 \pm 0.41$ & 20.49 & $<0.001$ \\
\hline ET-1 (umol/L) & $40.16 \pm 5.21$ & $44.81 \pm 6.33$ & $49.26 \pm 7.19$ & 8.657 & $<0.001$ \\
\hline
\end{tabular}

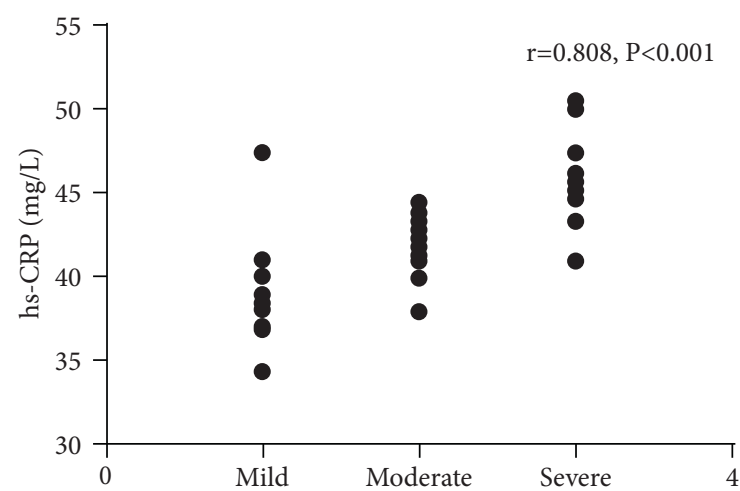

(a)

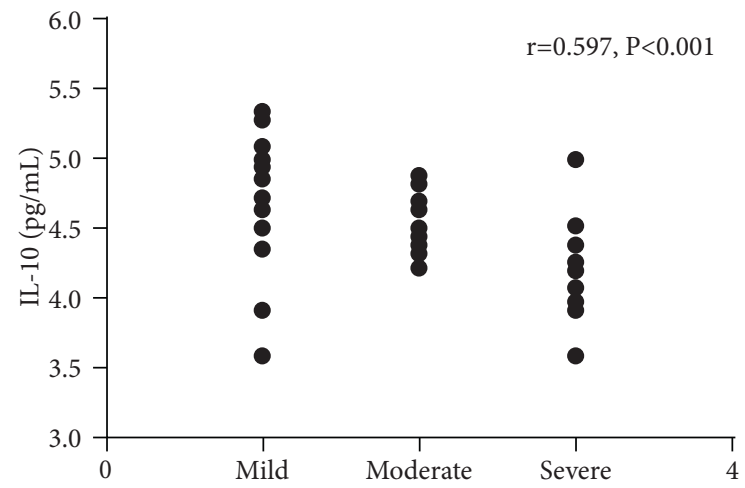

(c)

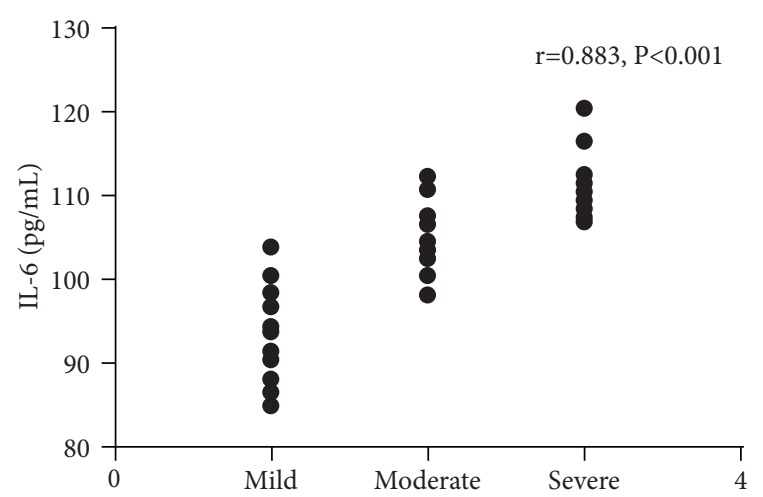

(b)

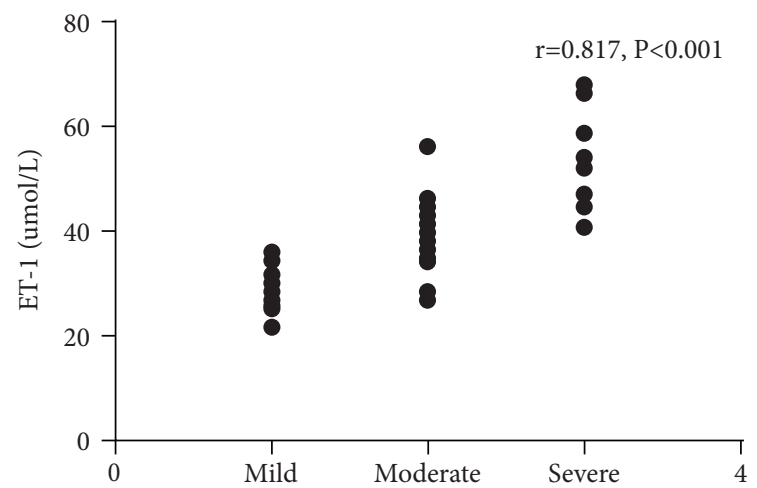

(d)

FIGURE 3: Correlation analysis of hs-CRP, IL-6, IL-10, ET-1, and COPD-PH severity. (a) Positive correlation between hs-CRP and severity of the disease $(r=0.808, P<0.05)$. (b) Positive correlation between IL-6 and severity of the disease $(r=0.883, P<0.03)$. (c) Negative correlation between IL-10 expression and severity of the disease $(r=-0.597, P<0.05)$. (d) Positive correlation between ET-1 and severity of the disease $(r=0.817, P<0.05)$.

\section{Discussion}

COPD is a respiratory disease characterized by restricted flow and damaged lung tissue [12]. $\mathrm{PH}$ is a disease characterized by the progressive rising of pulmonary vascular resistance. When COPD causes airway obstruction and damages pulmonary parenchyma and pulmonary vasculature, pulmonary arterial endothelium will suffer hyperplasia and fibrosis, eventually leading to pulmonary circulation remodeling and the onset of $\mathrm{PH}[13,14]$. $\mathrm{PH}$ in COPD patients is an irreversible pathological change, which has an important impact on the patient's poor prognosis [15].

A study pointed out that the incidence of COPD-PH is closely related to the inflammatory response [16], so the study explored the expression of several representative inflammatory factors of COPD-PH and their correlation with it. First, the two groups were compared in hs-CRP,
IL-6, IL-10, and ET-1 expression, and it turned out that the research group showed significantly higher serum hsCRP, IL-6, and ET-1 and significantly lower IL-10 expression than the control group, which indicated that COPD patients with $\mathrm{PH}$ and COPD patients without $\mathrm{PH}$ showed a significant difference in hs-CRP, IL-6, IL-10, and ET-1 expression and further suggested that the onset of COPD is related to systemic inflammatory response. hs-CRP, a typical sensitivity index of low-level inflammatory state produced by liver epithelial cells for stimulation by IL- 6 and other factors, can stimulate the release of inflammatory mediators such as ET-1 and further expand inflammatory response $[17,18]$. A study pointed out that ET-1, the most potent vasoconstrictor ever discovered, can promote lung vascular endothelium hyperplasia and fibrosis and aggravate pulmonary artery remodeling [19]. IL-6, a multifunctional 


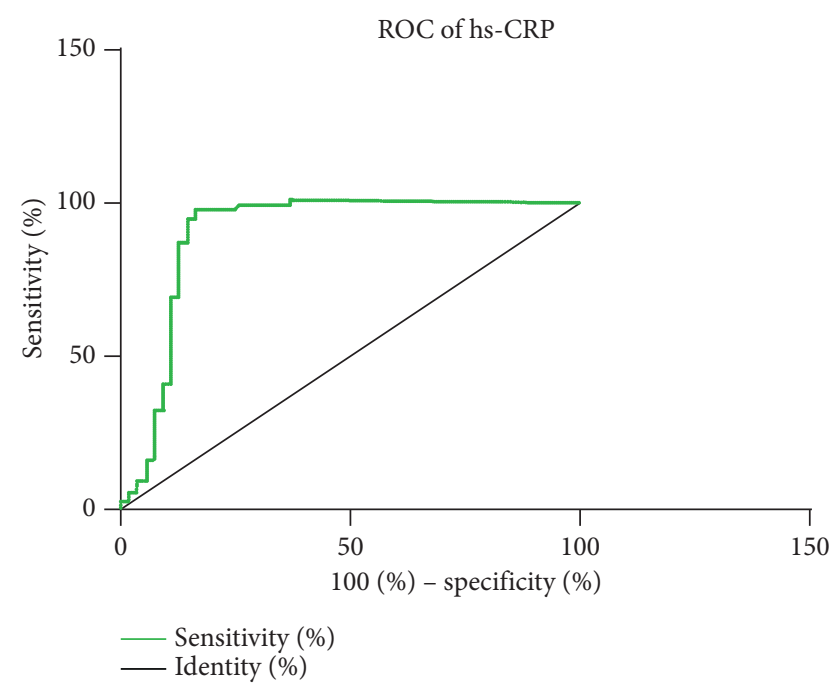

(a)

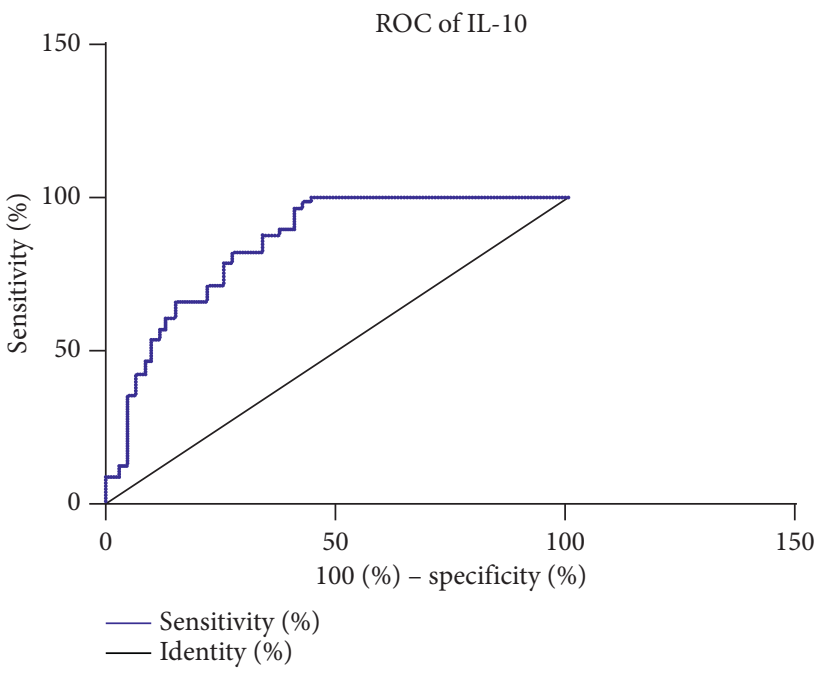

(c)

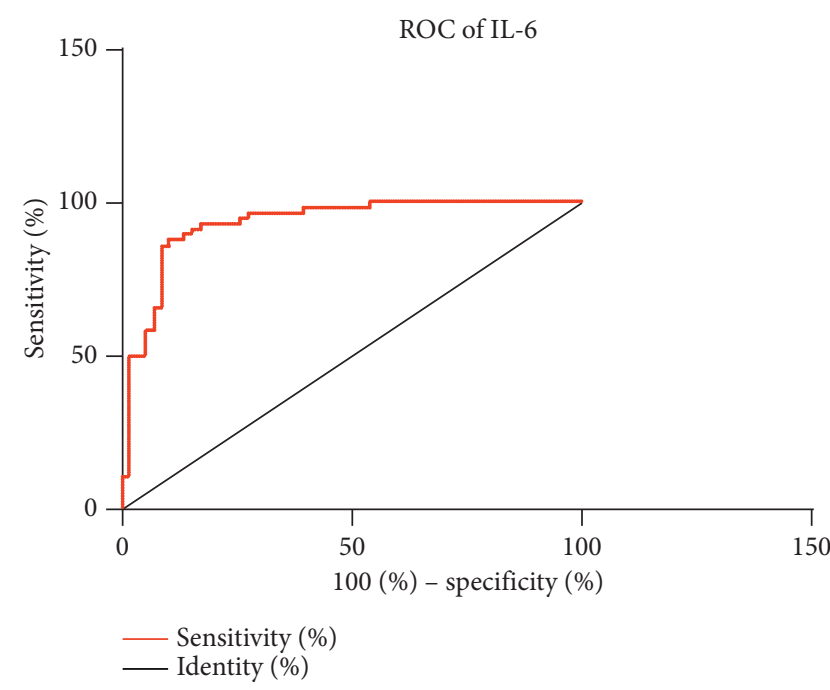

(b)

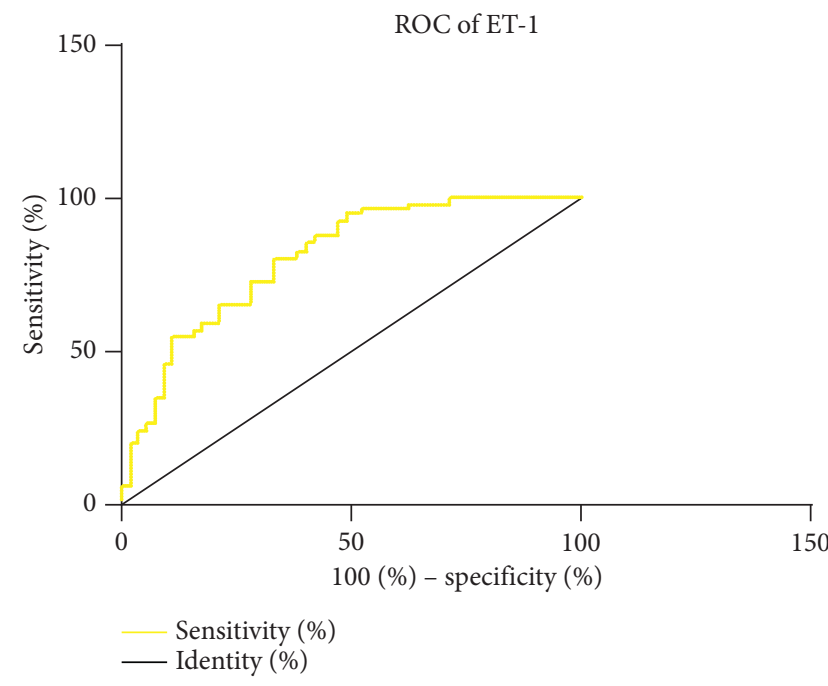

(d)

FIgURE 4: ROC analysis of hs-CRP, IL-6, IL-10, and ET-1 to COPD-PH. In terms of COPD-PH diagnosis, (a) hs-CRP shows sensitivity of $90.91 \%$, specificity of $85.96 \%$, AUG of 0.899 , and critical value of $38.49(\mathrm{mg} / \mathrm{L})$; (b) IL-6 shows sensitivity of $87.27 \%$, specificity of $89.47 \%$, AUG of 0.929 , and critical value of 98.99 (pg/mL); (c) IL-10 shows sensitivity of $81.82 \%$, specificity of $68.42 \%$, AUG of 0.854 , and critical value of $4.740(\mathrm{pg} / \mathrm{mL}$ ); (d) ET-1 shows sensitivity of $87.27 \%$, specificity of $57.89 \%$, AUG of 0.805 , and critical value of 39.64 (umol/L).

TABle 8: Assignment.

\begin{tabular}{lc}
\hline Items & Assignment \\
\hline hs-CRP & $\leq 38.49(\mathrm{mg} / \mathrm{L})=1,>38.49(\mathrm{mg} / \mathrm{L})=2$ \\
IL-6 & $\leq 98.99(\mathrm{pg} / \mathrm{mL})=1,>98.99(\mathrm{pg} / \mathrm{mL})=2$ \\
IL-10 & $\leq 4.740(\mathrm{pg} / \mathrm{mL})=1,>4.740(\mathrm{pg} / \mathrm{mL})=2$ \\
ET-1 & $\leq 39.64(\mathrm{umol} / \mathrm{L})=1,>39.64(\mathrm{umol} / \mathrm{L})=2$ \\
\hline
\end{tabular}

proinflammatory cytokine, plays a key role in the development of inflammatory response [20]. A study about hypoxia inducible mice pointed out that IL-6 involved in pulmonary inflammation and pulmonary vascular remodeling of mice and believed that L-6 involved in the effects of PH [21]. IL-10 is an anti-inflammatory factor that can inhibit the expansion of inflammatory response.
A study pointed out that COPD-PH patients have significantly lower serum IL-10 than the patients with COPD alone [22]. In addition, a study pointed out that increased pulmonary artery mechanical pressure will damage vascular endothelial cells, promote the release of inflammatory factors such as IL-6, and cause the liver to release hs-CRP through synthesis [23]. All of the above studies have confirmed and explained our experimental results.

In order to further explore the correlation between hs-CRP, IL-6, IL-10, ET-1, and PH, blood gas indexes and related indexes of lung function of the two groups were detected, and their correlations have been analyzed, and it turned out that serum hs-CRP, IL-6, and ET-1 were negatively correlated with $\mathrm{PaO} 2, \mathrm{FEV} 1, \mathrm{FVC}$, and FEV1/ FVC and positively related to $\mathrm{PaCO} 2$. It may be due to the 
TABLE 9: Multivariate analysis.

\begin{tabular}{lcccccc}
\hline Factors & $\beta$ & S.E & Wald & OR & $95 \%$ CI & $P$ \\
\hline hs-CRP & 2.793 & 0.425 & 1.983 & 2.314 & $1.104-4.211$ & $<0.01$ \\
IL-6 & 3.149 & 0.613 & 2.197 & 2.564 & $1.114-4.789$ & $<0.01$ \\
IL-10 & 3.169 & 0.642 & 2.231 & 6.151 & $3.119-5.243$ & $<0.01$ \\
ET-1 & 3.496 & 0.741 & 2.621 & 3.759 & $1.229-5.116$ & $<0.01$ \\
\hline
\end{tabular}

fact that inflammatory factors are involved in $\mathrm{PH}$ formation, and the formation will further lead to an increase in blood inflammatory factors of patients, causing a vicious circle. IL-10 was positively related to $\mathrm{PaO} 2, \mathrm{FEV} 1$, FVC, and FEV1/FVC and negatively elated to PaCO2. In addition, the correlation of serum hs-CRP, IL-6, IL-10, ET-1, and COPD-PH severity was analyzed, and it turned out that hs-CRP, IL-6, and ET-1 were positively correlated with COPD-PH severity, and IL-10 was negatively correlated with COPD-PH severity, which indicated that serum hs-CRP, IL-6, IL-10, and ET-1 were closely related to the severity of the disease and may be used as predictors for the disease.

In order to further analyze the influence of hs-CRP, IL-6, IL10 , and ET-1 on COPD-PH patients, ROC analysis was performed, and the results indicated that hs-CRP, IL-6, IL-10, and ET-1 have relatively high predictive value for COPD-PH. Then, assignment was performed to critical values, and logistic regression analysis was carried out. It indicated that elevation of hs-CRP, IL-6, and ET-1 and decrease of IL-10 were all independent risk factors for the onset of COPD-PH. It further suggested that inflammatory factors including hs-CRP, IL-6, IL10 , and ET-1 play important roles in the onset and progression of COPD-PH. A study about the influence of inflammatory factors on COPD-PH pointed out that IL- 6 and CRP may be independent risk factors for COPD-PH, which is consistent with some of our results [24]. Direct determination of $\mathrm{PH}$ in COPD is traumatic, so it is of great clinical significance to detect hs-CRP, IL-6, IL-10, and ET-1 to monitor pulmonary hypertension [25].

\section{Conclusion}

In conclusion, hs-CRP, IL-6, IL-10, and ET-1 were closely and significantly related to the pathological process of COPD-PH, including onset and development, and the elevation of hs-CRP, IL-6, and ET-1 and decrease of IL-10 are independent risk factors for the onset of COPD-PH. With relatively high predictive value for COPD-PH, hs-CRP, IL-6, IL-10, and ET-1 can be promoted as predictors for it. There are certain shortcomings of this study. For example, the diagnostic value of hs-CRP, IL-6, IL-10, and ET-1 for COPD-PH severity was not explored, and because of limited number of samples included, the conclusion may be exploratory. In the subsequent research, we will include more samples and supplement the details of the experiment to make the conclusion more substantial.

\section{Data Availability}

The datasets used and/or analyzed during the current study are available from the corresponding author upon request.

\section{Conflicts of Interest}

The authors declare that they have no conflicts of interest.

\section{References}

[1] L. V. Wain, N. Shrine, N. Shrine et al., "Genome-wide association analyses for lung function and chronic obstructive pulmonary disease identify new loci and potential druggable targets," Nature Genetics, vol. 49, no. 3, pp. 416-425, 2017.

[2] A. A. Bajwa, A. Shujaat, M. Patel, C. Thomas, F. Rahaghi, and C. D. Burger, "The safety and tolerability of inhaled treprostinil in patients with pulmonary hypertension and chronic obstructive pulmonary disease," Pulmonary Circulation, vol. 7, no. 1, pp. 82-88, 2017.

[3] R. Ffrench-O'Carroll, B. Biancardi, and O. Tujjar, "Malar rash with pulmonary hypertension and chronic obstructive pulmonary disease," BMJ Case Reports, vol. 2017, Article ID 220589, 2017.

[4] L. Wang, Y.-Z. Jin, Q.-H. Zhao et al., "Hemodynamic and gas exchange effects of inhaled iloprost in patients with COPD and pulmonary hypertension," International Journal of Chronic Obstructive Pulmonary Disease, vol. Volume 12, pp. 3353-3360, 2017.

[5] A. Chaouat, L. Savale, C. Chouaid et al., "Role for interleukin6 in COPD-related pulmonary hypertension," Chest, vol. 136, no. 3, pp. 678-687, 2009.

[6] N. Liu, S. Parry, Y. Xiao, S. Zhou, and Q. Liu, "Molecular targets of the Warburg effect and inflammatory cytokines in the pathogenesis of pulmonary artery hypertension," Clinica Chimica Acta, vol. 466, pp. 98-104, 2017.

[7] R. Ferrari, S. E. Tanni, L. M. Caram, C. Corrêa, C. R. Corrêa, and I. Godoy, "Three-year fol-low-up of interleukin 6 and C-reactive protein in chronic ob-structive pulmonary disease," Respiratory Research, vol. 14, no. 24, pp. 234-241, 2013.

[8] M. Jasiewicz, M. Knapp, E. Waszkiewicz et al., "Enhanced IL-6 trans-signaling in pulmonary arterial hypertension and its potential role in disease-related systemic damage," Cytokine, vol. 76, no. 2, pp. 187-192, 2015.

[9] W. L. Hsu, Y. C. Lin, J. R. Jeng, H. Y. Chang, and T. C. Chou, "Baicalein ameliorates pulmonary arterial hypertension caused by monocrotaline through downregulation of ET-1 and ETAR in pneumonectomized rats[J]," American Journal of Chinese Medicine, vol. 46, no. 4, p. 1, 2018.

[10] A. H. Chester and M. H. Yacoub, "The role of endothelin-1 in pulmonary arterial hypertension[J]," Glob Cardiol Sci Pract, vol. 2014, no. 2, pp. 62-78, 2017.

[11] M. R. Fisher, G. J. Criner, A. P. Fishman et al., "Estimating pulmonary artery pressures by echocardiography in patients with emphysema," European Respiratory Journal, vol. 30, no. 5, pp. 914-921, 2007.

[12] D. Koulenti, F. R. Parisella, E. Xu, J. Lipman, and J. Rello, "The relationship between ventilator-associated pneumonia and chronic obstructive pulmonary disease: what is the current evidence?" European Journal of Clinical Microbiology \& Infectious Diseases, vol. 38, no. 4, pp. 637-647, 2019.

[13] K. Ando, H. Kuraishi, T. Nagaoka et al., "Potential role of CT metrics in chronic obstructive pulmonary disease with pulmonary hypertension," Lung, vol. 193, no. 6, pp. 911-918, 2015.

[14] N. Tanabe, H. Taniguchi, I. Tsujino et al., "Multi-institutional retrospective cohort study of patients with severe pulmonary hypertension associated with respiratory diseases," Respirology, vol. 20, no. 5, pp. 805-812, 2015. 
[15] S. Yano, K. Kobayashi, K. Kato, and T. Ikeda, "The study of pulmonary hypertension and plasma BNP values in respiratory diseases," Japanese Respiratory Society, vol. 44, no. 2, pp. 99-103, 2006.

[16] W. Cornwell, V. Kim, C. Song, and T. Rogers, "Pathogenesis of inflammation and repair in advanced COPD," Seminars in Respiratory and Critical Care Medicine, vol. 31, no. 03, pp. 257-266, 2010.

[17] H. Ghobadi, N. Fouladi, K. Beukaghazadeh, and K. Ansarin, "Association of high sensitive CRP level and COPD assessment test scores with clinically important predictive outcomes in stable COPD patients[J]," Tanaffos, vol. 14, no. 1, pp. 34-41, 2015.

[18] A. N. Nillawar, K. B. Joshi, S. B. Patil, J. S. Bardapurkar, and S. J. Bardapurkar, "Evaluation of HS-CRP and lipid profile in COPD," Journal of Clinical \& Diagnostic Research Jcdr, vol. 7, no. 5, pp. 801-803, 2013.

[19] J. A. Mazurek, T. Jabuonski, and R. Zolty, "Endothelin-1 levels in COPD-induced pulmonary hypertension," Journal of Cardiac Failure, vol. 18, no. 8, p. S29, 2012.

[20] M. A. Ghebre, P. H. Pang, S. Diver et al., "Biological exacerbation clusters demonstrate asthma and chronic obstructive pulmonary disease overlap with distinct mediator and microbiome profiles," The Journal of Allergy and Clinical Immunology, vol. 141, no. 6, pp. 2027-2036, 2018, e12.

[21] L. Savale, L. Tu, D. Rideau et al., "Impact of interleukin-6 on hypoxia-induced pulmonary hypertension and lung inflammation in mice," Respiratory Research, vol. 10, no. 2, pp. 1-13, 2009.

[22] G. Paone, V. Conti, G. Biondi-Zoccai et al., "Long-term home noninvasive mechanical ventilation increases systemic inflammatory response in chronic obstructive pulmonary disease: a prospective observational study," Mediators of Inflammation, vol. 2014, no. 22, Article ID 503145, 2015.

[23] A. D’Alessandro, K. C. El Kasmi, L. Plecitá-Hlavatá et al., "Hallmarks of pulmonary hypertension: $\mathrm{m}$ and inflammatory cell metabolic reprogramming," Antioxidants and Redox Signaling, vol. 28, no. 3, pp. 230-250, 2018.

[24] G. Weidong, J. Chengyang, and L. Cuiying, "Correlation between inflammatory response factors and pulmonary hypertension in patients with chronicobstructive pulmonary disease," Chinese Medical Record, vol. 19, no. 9, 2018.

[25] A. Machraoui, A. Helfen, D. S. Von, D. Schött, W. T. Ulmer, and J. Barmeyer, "Diagnostic value of right thoracic ECG recording in detection of pulmonary hypertension in chronic obstructive respiratory disease," Herz, vol. 19, no. 19, pp. 182-188, 1994. 